\title{
Primary cardiac tumors: a spectrum of pathologies and scenarios
}

\author{
Mohammed Sanad ${ }^{1,2,3^{*}}$ (D) Sherif Arafa ${ }^{4}$, Mohammed Adel Hegazy ${ }^{5}$ and Wael Abdel Aziz Abdel Hamid ${ }^{1,2}$
}

\begin{abstract}
Background: Cardiac tumors are rare, and their diagnosis is challenging. The level of evidence for the management of cardiac tumors came from case reports or small case series. We aim to present our multicenter experience in the diagnosis and management of cardiac tumors.

Results: We performed a prospective study, including 82 cases of cardiac tumors encountered at three centers in 5 years. The mean age was $62 \pm 5.8$ years old (range 2 months-74 years), and $91.4 \%$ were females constituted. The median EuroSCORE II was 4.27\%. All cases underwent radical excision of the tumor. Five cases underwent additional mitral valve replacement and one case underwent coronary bypass grafting. Pathological diagnoses were left atrial myxomas $(n=65,79.3 \%)$, right atrial myxomas $(n=5,6.1 \%)$, bi-atrial myxomas $(n=2,2.4 \%)$, right ventricular outflow tract myxoma $(n=1,1.2 \%)$, myxosarcoma $(n=1,1.2 \%)$, thymoma $(n=1,1.2 \%)$, recurrent hemangioendothelioma $(n=1,1.2 \%)$, round cell tumor $(n=1,1.2 \%)$, cardiac lymphoma $(n=1,1.2 \%)$, rhabdomyomas $(n=2,2.4 \%)$, and renal cell carcinomas $(n=2,2.4 \%)$. Two patients (2.4\%) had re-exploration for bleeding. One patient (1.2\%) with right atrial thymoma experienced a severe vasoplegia with unstable hemodynamics. Two patients had operative mortality (2.4\%), one with recurrent cardiac hemangioendothelioma. During 1-year follow-up, one patient (1.2\%) died because of metastatic myosarcoma. Recurrence was reported in 2 patients (2.4\%).

Conclusions: The clinical scenario of cardiac tumors depends mainly on tumor location and size rather than the histopathology. Surgical resection is feasible in benign tumors while very challenging in malignant tumors.

Keywords: Cardiac tumor, Malignancy, Cardiac lesion, Survival
\end{abstract}

\section{Background}

Cardiac tumors are rare, with an incidence of less than $0.33 \%$ [1]. They are categorized as primary and secondary, depending on the origin of the tumors. Primary cardiac tumors present $5 \%$ of all cardiac tumors and originate primarily from the heart. In contrast, secondary cardiac tumors are the most common cardiac tumors, and they present metastasis to the heart from other organs [1].

Around $75 \%$ of primary tumors are benign, and $75 \%$ of these are atrial myxomas $[1,2]$.

\footnotetext{
* Correspondence: mohamedsanad2008@yahoo.com; mams@mans.edu.eg 'Department of Cardiothoracic Surgery, Faculty of Medicine, Mansoura University, Mansoura, Dakahlia, Egypt

${ }^{2}$ Department of Cardiothoracic Surgery, Nasser Institute for Research and

Treatment, Egypt Ministry of Health and Population, Cairo, Egypt

Full list of author information is available at the end of the article
}

Myxomas commonly originate from the left atrium, and ventricular myxomas were rarely reported. Additionally, infantile myxoma was not frequently reported in the literature [3].

Surgical excision is usually curative in primary cardiac tumors, and it is the main treatment modality. In secondary tumors, surgery has a palliative role in symptoms relief $[2,3]$.

Currently, the level of evidence for the management of cardiac tumors is derived from case reports or small case series, and specific tumors were rarely presented in the literature. This study aims to review our multicenter experience in the diagnosis and management of primary cardiac tumors. 


\section{Methods}

\section{Study design and setting}

This prospective multicentric cohort study included a total of 82 consecutive patients operated for primary cardiac tumors at three referral centers from 2013 to 2018. All cases had a 1-year follow-up.

\section{Inclusion and exclusion criteria}

All cases with primary cardiac tumors with no evidence of metastasis at the different age groups were included in our study. Cases with secondary cardiac tumors were excluded.

One hundred seven patients with cardiac tumors were examined for eligibility criteria. We excluded 25 patients with secondary cardiac tumors, and a total of 82 eligible patients diagnosed with primary cardiac tumors were enrolled.

\section{Ethical considerations}

We conducted the study according to the declaration of Helsinki. Approvals of the Institutional Research Boards were obtained. Informed written consent was obtained from all cases after the explanation of the details and possible complications of the surgical intervention. All patients signed written consent for publishing the medical data without identification.

\section{Surgical workup and approach}

All cases were subjected to complete history taking, thorough physical examination, and routine preoperative laboratory investigations. Besides, radiological investigations including chest X-ray and echocardiography (transthoracic and/or transesophageal) were performed to assess the site, size, and tumor extension. In all malignant tumors, a metastatic workup was performed preoperatively.

All cases presenting with cardiac tumors were operated on emergency basis for fear of complications of the intracardiac mass as per policy of our centers.

The cardiac anesthesia team performed full invasive monitoring, and general anesthesia was used in all cases as per standard of care.

Following tailored surgical planning, all cases were operated immediately after diagnosis through a full median sternotomy. Cardiopulmonary bypass was conducted using aortic and bicaval cannulation with moderate hypothermia, and intermittent antegrade cold blood cardioplegia was used in all cases. The incision depends on the location of the tumor, and care was taken to prevent the fragmentation of the masses. Concomitant mitral valve replacement was performed in five cases. Irrigation and suctioning of the atria and ventricles were performed to ensure no residual fragmentations. Following cardiac reconstruction, weaning off bypass was performed, and the surgery was completed as standard.

\section{Postoperative management}

Following the operation, the cases were transferred to the cardiac surgery intensive care unit (ICU) under full invasive monitoring, then to the ward unless complications occurred. Early ambulation and chest physiotherapy were encouraged. Pain control was achieved using non-steroidal anti-inflammatory drugs (NSAIDs) and opioids accordingly. Immediate postoperative echocardiography was performed to all patients.

All cases were reviewed for the need for postoperative adjuvant therapy by the multidisciplinary team (MDT). At the regular follow-up visits, cases were assessed clinically and radiologically for the outcome of resection.

\section{Study outcomes}

We reported the histological types of the resected specimens and the postoperative morbidity and mortality.

\section{Statistical analysis}

The data were tabulated and analyzed using the IBM SPSS software version 22.0 (IBM Inc., Chicago, IL, USA). Qualitative data were described using the number and percent. Quantitative variables were reported as mean and standard deviation (SD).

\section{Results}

\section{Demographic data and surgical findings}

The mean age was $62 \pm 5.8$ years old (range 2 months74 years), and 75 patients were females (91.4\%). All cases underwent radical excision of the tumors. Five cases underwent concomitant mitral valve replacement with a hemangioendothelioma, a myxosarcoma, and three myxomas excisions. One case was operated for left atrial myxoma, single-vessel coronary artery bypass grafting, along with mitral valve replacement and tricuspid valve annuloplasty. The preoperative demographic and clinical data are mentioned in Table 1. The operative data are presented in Table 2. The pathology of the operated cases is shown in Table 3.

\section{Morbidity and mortality}

Two patients had re-exploration for bleeding. One patient with chronic viral hepatitis experienced high drainage that was controlled with blood product transfusion. No patient had complete heart block, and one patient with infiltrating cardiac hemangioendothelioma had an incomplete heart block that was managed with temporary epicardial pacing and was weaned on the second postoperative day. One patient was diagnosed with right atrial thymoma and experienced severe vasoplegia and unstable hemodynamics. 
Table 1 Preoperative characteristics of the study patients

\begin{tabular}{|c|c|c|}
\hline \multicolumn{2}{|l|}{ Variables } & $n=82$ \\
\hline \multicolumn{2}{|l|}{ Age (years) } & $62 \pm 5.8$ (2 months -74 years) \\
\hline \multicolumn{2}{|l|}{ Females } & $75(91.46)$ \\
\hline \multicolumn{2}{|l|}{ Body mass index $\left(\mathrm{kg} / \mathrm{m}^{2}\right)$} & $27.8 \pm 4.3(16.6-44.1)$ \\
\hline \multicolumn{2}{|l|}{ Smoking status (ex-smoker or current smoking) } & $12(14.63)$ \\
\hline \multicolumn{2}{|l|}{ EuroSCORE ॥ } & $4.27(2.39-56.37)$ \\
\hline \multicolumn{2}{|l|}{ Extracardiac arteriopathy } & 0 \\
\hline \multicolumn{2}{|l|}{ Poor mobility } & 0 \\
\hline \multicolumn{2}{|l|}{ Diabetes mellitus (insulin-dependent) } & $24(29.26)$ \\
\hline \multicolumn{2}{|l|}{ Hypertension } & $38(46.34)$ \\
\hline \multicolumn{2}{|l|}{ Dyslipidemia } & $24(29.27)$ \\
\hline \multicolumn{2}{|l|}{ Viral hepatitis C } & $12(14.63)$ \\
\hline \multicolumn{2}{|l|}{ Ischemic heart disease } & $2(2.44)$ \\
\hline \multicolumn{2}{|l|}{ Active endocarditis } & 0 \\
\hline \multicolumn{2}{|l|}{ Prior cardiac surgery } & 0 \\
\hline \multicolumn{2}{|l|}{ Chronic lung disease } & $16(19.52)$ \\
\hline \multicolumn{2}{|l|}{ Critical preoperative condition } & $6(7.3)$ \\
\hline \multirow[t]{4}{*}{ Functional classification } & NYHA I & $5(6.1)$ \\
\hline & NYHA II & $3(3.65)$ \\
\hline & NYHA III & $68(82.92)$ \\
\hline & NYHA IV & $6(7.3)$ \\
\hline \multirow[t]{4}{*}{ Left ventricular function } & $E F>50 \%$ & $79(96.34)$ \\
\hline & EF $31-50 \%$ & $2(2.44)$ \\
\hline & EF $21-30 \%$ & $1(1.22)$ \\
\hline & $E F<21 \%$ & 0 \\
\hline \multicolumn{2}{|l|}{ Pulmonary hypertension } & $38(46.43)$ \\
\hline \multirow[t]{4}{*}{ Presenting symptom } & Asymptomatic & $10(12.19)$ \\
\hline & Syncopal attacks & $16(19.52)$ \\
\hline & Shortness of breath & $72(87.8)$ \\
\hline & Embolic complications & $3(3.65)$ \\
\hline
\end{tabular}

$S D$ standard deviation, $n$ number of patients, EuroSCORE European system for cardiac operative risk evaluation, NYHA New York Heart Association, EF ejection fraction, $C H D$ coronary heart disease. (Continuous data are presented as mean and standard deviation and categorical data as number and percent)

Two patients had operative mortality, and one had late postoperative morality. One patient was a 35-year-old male with recurrent cardiac hemangioendothelioma with chest wall invasion and mediastinal lymphadenopathy, and mortality was encountered on the 2 nd postoperative day.

The second case was a 52-year-old female with ischemic heart disease (IHD), left atrial myxoma with severe mitral valve stenosis (MS), severe tricuspid valve regurgitation (TR), and poor biventricular function with $25 \%$ ejection fraction. She underwent radical excision of the mass, mitral valve replacement (MVR), De-Vega tricuspid valve annuloplasty (TVR), and single-vessel coronary artery bypass grafting (CABG) (saphenous vein graft to an obtuse marginal artery). The patient had long cross- clamp (150 min) and bypass times (260 min). Weaning off bypass failed. Pathology confirmed the diagnosis of myxoma.

The third was late postoperative mortality in a $42-$ year-old female patient who underwent resection for myxosarcoma. She presented 3 months later with recurrence in the chest wall, and she died after 1 week in the oncology department. No other mortalities were recorded upon a during 1-year follow-up.

\section{Scenarios of rare cases Recurrent cardiac hemangioendothelioma}

A 35-year-old male patient presented with a 3-month history of progressive dyspnea. 
Table 2 Intraoperative characteristics of the study patients

\begin{tabular}{|c|c|c|}
\hline Variables & & $n=82$ \\
\hline \multirow[t]{4}{*}{ Priority of surgery } & Elective & $3(3.66)$ \\
\hline & Urgent & $7(8.54)$ \\
\hline & Emergency & $71(86.58)$ \\
\hline & Salvage & $1(1.2)$ \\
\hline \multirow[t]{5}{*}{ Site of the tumor } & Left atrium & $68(82.92)$ \\
\hline & Right atrium & $11(13.41)$ \\
\hline & Biatrial & $2(2.44)$ \\
\hline & Right ventricular outflow tract & $1(1.22)$ \\
\hline & Inferior vena cava & $2(2.44)$ \\
\hline \multirow[t]{5}{*}{ Surgical approach } & Biatriotomy & $34(41.46)$ \\
\hline & Left atriotomy & $20(24.39)$ \\
\hline & Right atriotomy & $26(31.71)$ \\
\hline & Right ventriculotomy & $1(1.22)$ \\
\hline & Aortotomy & $1(1.22)$ \\
\hline \multirow[t]{3}{*}{ Complexity of procedure } & Isolated & $76(92.69)$ \\
\hline & Two procedures & $5(6.09)$ \\
\hline & Three procedures & $1(1.22)$ \\
\hline \multirow[t]{3}{*}{ Type of surgery } & Tumor excision & $76(92.69)$ \\
\hline & Excision and MVR & $5(6.09)$ \\
\hline & Excision, single vessel CABG, MVR, TVR & $1(1.22)$ \\
\hline
\end{tabular}

$C A B G$ coronary artery bypass grafting, MVR mitral valve replacement, $T V R$ tricuspid valve repair. (categorical data as number and percent)

Transesophageal echocardiography (TEE) (Fig. 1a, b) revealed an intracardiac mass measuring $2.2 \times 5 \mathrm{~cm}$, infiltrating the lower $1 / 3$ of the interatrial septum (IAS), with direct extension to the atrial surface of the anterior mitral leaflet (AML) till its tips for $5.5 \mathrm{~cm}$ distance with proper differentiation between the mass and the chordal tissue with good biventricular function.

Intraoperatively, left atriotomy revealed a large tumor that was not separable from the mitral valve. The tumor was extending through the mitral valve annulus.

Table 3 Pathological diagnoses of the studied patients

\begin{tabular}{ll}
\hline Pathology & $n(\%)$ \\
\hline Right atrial myxoma & $5(6.1)$ \\
Left atrial myxoma & $65(79.27)$ \\
Biatrial myxoma & $2(2.44)$ \\
RVOT myxoma & $1(1.22)$ \\
Myxosarcoma & $1(1.22)$ \\
Thymoma & $1(1.22)$ \\
Hemangioendothelioma & $1(1.22)$ \\
Round cell tumor & $1(1.22)$ \\
Cardiac lymphoma & $1(1.22)$ \\
Rhabdomyoma & $2(2.44)$ \\
Tumor extension from RCC & $2(2.44)$ \\
\hline
\end{tabular}

RVOT right ventricular outflow tract, $R C C$ renal cell carcinoma. (Categorical data as number and percent)
The decision was radical excision. The fossa ovalis was excised with all the tumor tissue, which was extending into the lower part of the septum till the IVC and till the muscular apical part of the IAS. The AML with the tumor was excised totally except a tiny small part near the site of the left circumflex (LCX) artery. MVR was performed after creating a new mitral valve annulus using polypropylene suture 4/0. A mechanical valve of size 25 was inserted. IAS was closed using a pericardial patch, and TV repair was done using DeVaga semicircular tricuspid annuloplasty. The patient had an incomplete heart block that was managed using temporary pacing. ICU stay was 5 days, and the hospital stay was 7 days.

Histopathological sections revealed a vascular tumor, and the vessels were arranged in hemangiopericytoma pattern. Immunohistochemistry was positive for CD34 and negative for CD99, desmin, and myogenin. The diagnosis was consistent with hemangioendothelioma.

The oncologist recommended no adjuvant chemo/ radiotherapy. Upon close follow-up, the patient presented 10 months later with a recurrence of symptoms. A workup was done including echocardiography, contrast computed tomography $(\mathrm{CT})$, cardiac magnetic resonance imaging (CMRI), and ${ }^{18} \mathrm{~F}$-FDG positron emission tomography (PET-CT) (Fig. 1c, d).

Workup revealed a large, irregular, heterogeneous, lobulated intracardiac mass involving the IAS, creeping 


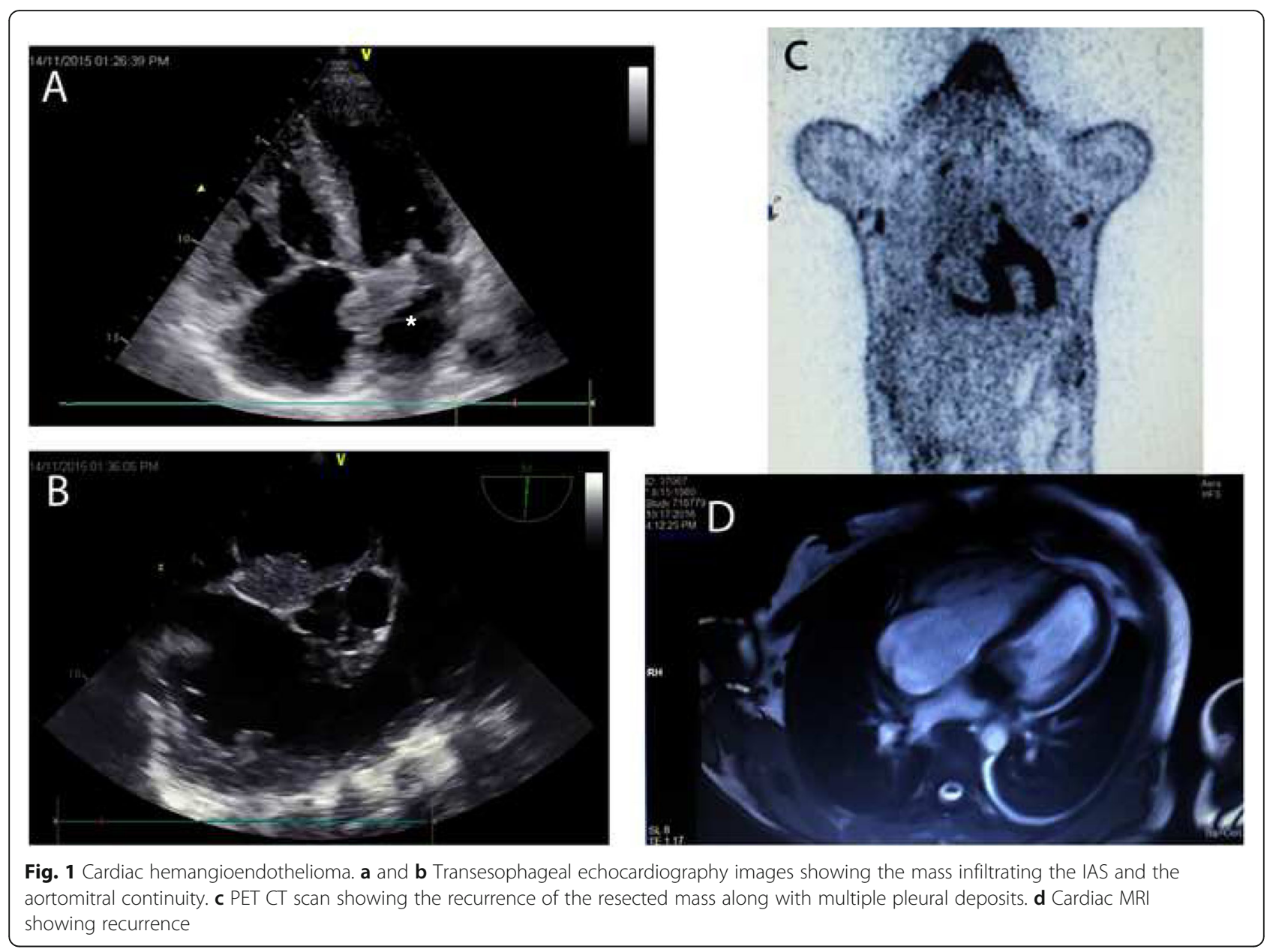

through the mitral inflow towards the left ventricular outflow tract (LVOT) and the aortic root. The lesion was protruding through the left atrium (LA) and left ventricular (LV) lumens. It measured $6.2 \mathrm{~cm}$ and had a maximum standard uptake value (SUVmax of 14.3). The lesion was expanding bilaterally to the costochondral junctions. Multiple axillary and superior mediastinal, prevascular, para-tracheal, and aortopulmonary lymphadenopathy were noted. Bilateral parietal pleural nodules were seen with SUVmax of 2.9. The right internal mammary artery was involved with a 1.4- $\mathrm{cm}$ lesion with a SUVmax of 5.8 .

The patient underwent a re-operative surgery for recurrence. The tumor was attached to the annulus and the interventricular septum (IVS) and obstructing the LVOT; therefore, a biopsy was taken. Mortality occurred on the 2 nd postoperative day.

\section{Right atrial thymoma}

A 70-year-old male had right atrial mass measuring $10 \times$ $6 \mathrm{~cm}$ by echocardiography. Radical resection of the mass was performed. The specimen is exhibited in (Fig. 2). Histopathological assessment was consistent with right atrial thymoma. The postoperative course was stormy, and the patient developed vasoplegia postoperatively that was managed with intravenous (IV) methylene blue injection. The patient was discharged on the 10th postoperative day. No recurrence was recorded upon 1 year of follow-up.

\section{Infantile right atrial myxoma}

A 2-month-old boy presented with cyanosis, tachypnea, and systemic congestion. Echocardiography showed a mass in the right atrium obstructing the tricuspid valve. The excision of the mass was done along with the right atrial wall attachment (Fig. 3). Reconstruction of the IAS and the right atrial wall was done using autologous pericardium. Histopathology was consistent with right atrial myxoma. No recurrence was recorded upon 1 year of follow-up.

\section{Left atrial lymphoma}

A 65-year-old female patient presented with dyspnea NYHA functional class III for 3 months. Echocardiography revealed a left atrial mass. Upon left atriotomy, the mass was attached to the free will of the left atrium 


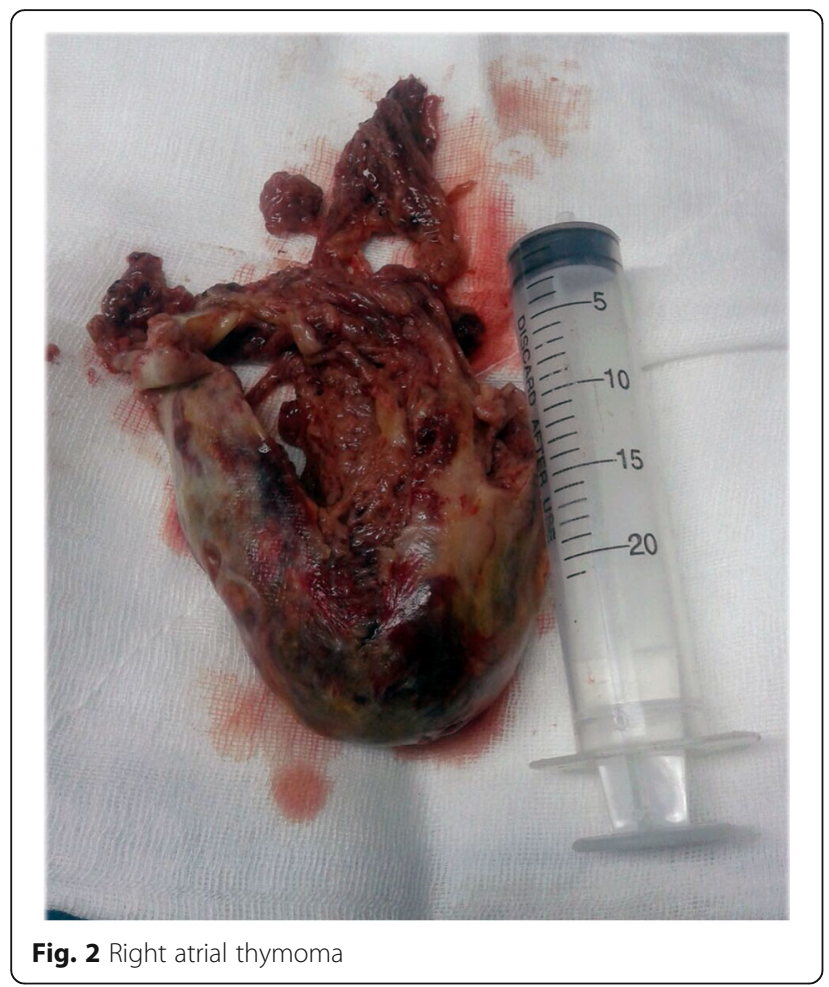

near the mitral annulus, and radical excision was performed. Gross examination exhibited a tan-colored fleshy tissue mass measuring about $4.5 \times 3 \times 5 \mathrm{~cm}$ (Fig. 4). Histopathology revealed extensive necrotic tumor tissue with viable areas of malignant tumor infiltration. Immunohistochemistry (IHC) showed positive diffuse membranous staining of malignant cells for LCA, CD20\& cytoplasmic stain for vimentin and negative for desmin, S100, CD34, CD3, and CD99. The diagnosis was consistent with intracardiac left atrial non-Hodgkin's lymphoma, diffuse large beta-cell lymphoma (NHL-DLBCL) proved by positivity for MUM1, BCL6, and BCL2 and negativity for CD10.

Following the decision of the MDT, the patient received postoperative chemotherapy. She was followed up for 1 year without recurrence before she was lost to follow up.

\section{Right ventricular outflow tract (RVOT) myxoma}

A 41-year-old female presented with lower limb edema and systemic congestion symptoms. Echocardiography showed a right ventricular mass obstructing the RVOT. Right atriotomy and right ventriculotomy were done to excise the mass attached to the interventricular septum (IVS) obstructing the RVOT (Fig. 5a, b, c). An 8-cm right ventriculotomy incision was performed at the infundibulum of the RVOT $2 \mathrm{~cm}$ lateral to the left anterior descending coronary artery near to the apex. The mass was assessed through the right ventriculotomy incision. The basal attachment was found originating from the
IVS. The specimen was dissected and then excised from the IVS. Pathology was consistent with RVOT myxoma.

\section{Discussion}

The incidence of primary cardiac tumors varies between 0.001 and $0.03 \%$, and $0.3-0.85 \%$ of all open-heart surgeries are for cardiac tumor resections $[4,5]$. Twenty-five percent of primary cardiac tumors are malignant, with sarcoma accounting for a majority of the reported cases [6]. Most of the primary cardiac tumors reported in our series of 82 patients were benign (91\%), and atrial myxoma was the most common type (87\%). Kumar and associates reported a $98 \%$ prevalence of primary cardiac tumors in their series, and $92 \%$ of them were benign [4].

Nomoto and coworkers [7] reported a prevalence of $64 \%$ for primary cardiac tumors in 61 patients in a 15 year prospective study. The reported mean age was 65 years. Benign tumors were found in 30 cases out of 41 patients who had surgery. Myxoma (67\%) was the most common type, followed by papillary fibroelastoma (23\%). The most common site was the left atrium (35\%), followed by the right atrium (25\%). Primary malignant tumors were diagnosed in 10 cases (24\%), including six angiosarcomas, three lymphomas, and one leiomyosarcoma [7]..

Cardiac tumors yield symptoms through any of 4 mechanisms: obstruction of blood flow, local invasion inducing arrhythmias, systemic or pulmonary embolization [2, 5, 7], and systemic or constitutional symptoms $[5,7]$.

Embolization was reported in $30-43 \%$ of these patients [5]. None of our cases had thromboembolism postoperatively, which could be attributed to the rapid intervention once the diagnosis is established. However, three patients presented with a prior history of thromboembolism, two right-sided strokes, and one right-sided lower limb ischemia.

Our patients presented mainly with dyspnea (87.8\%), followed by syncopal attacks (19.52\%) in cases with left atrial masses causing mitral valve obstruction. Ten patients (12.19\%) were asymptomatic. Chest pain proposes malignancy in nearly all cases, but angina can result from coronary artery tumor embolism in either benign or malignant tumors [7]. All patients in our series were operated upon on an emergency basis once the diagnosis has been established.

Echocardiography was the first diagnostic modality, and further imaging was rarely required in our patients. 18F-fluorodeoxyglucose positron emission tomography/ computed tomography (FDG-PET/CT) has a sensitivity of over $90 \%$ in differentiating benign and malignant processes [8]. In our study, one case with a recurrent hemangioendothelioma required both FDG-PET/CT and CMR to assess the extent of recurrence and metastasis. 

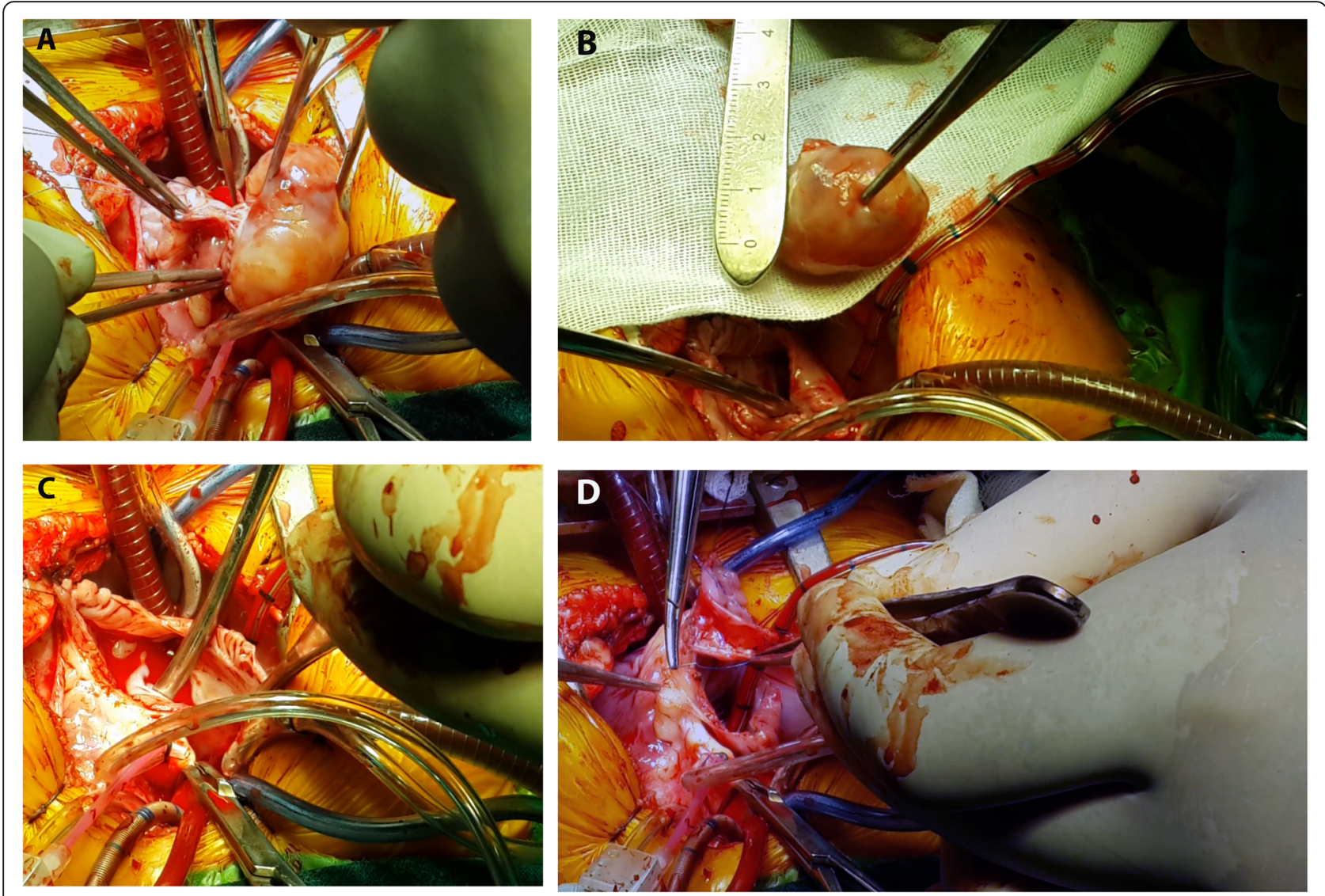

Fig. 3 Infantile right atrial myxoma. a The mass is observed attached to the IAS and excised with a free margin of the IAS and the right atrial wall. b Specimen after excision showing a mass of size $4 \times 2 \mathrm{~cm}$. c The defect in the IAS. D Reconstruction of the IAS using autologous pericardium and closure of the right atrial wall

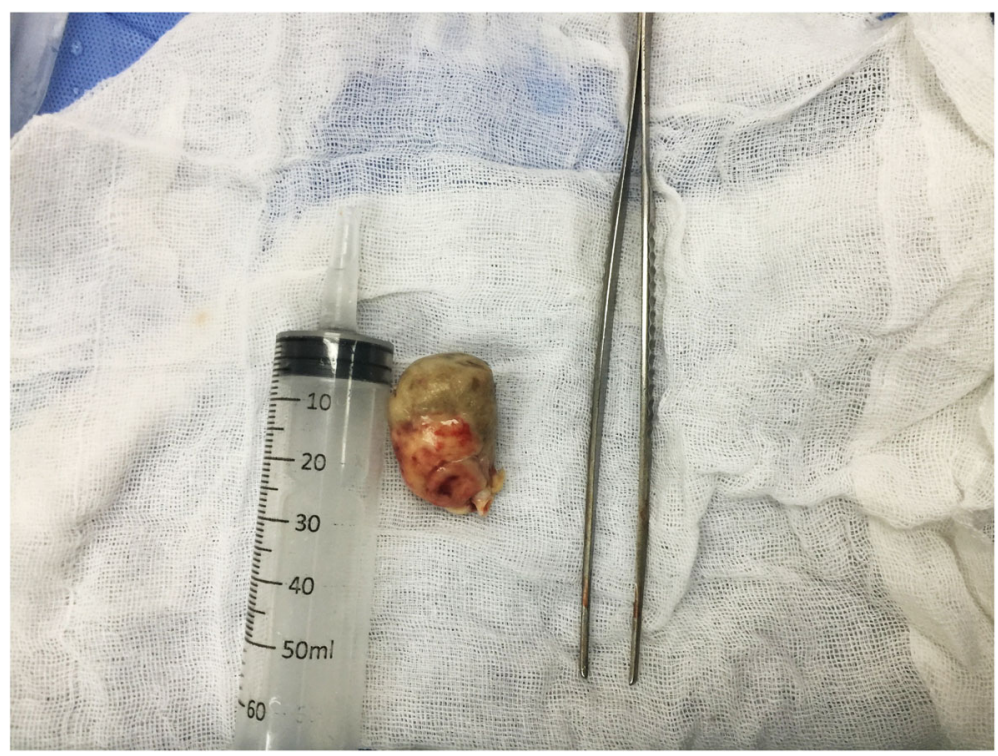

Fig. 4 Intracardiac lymphoma 

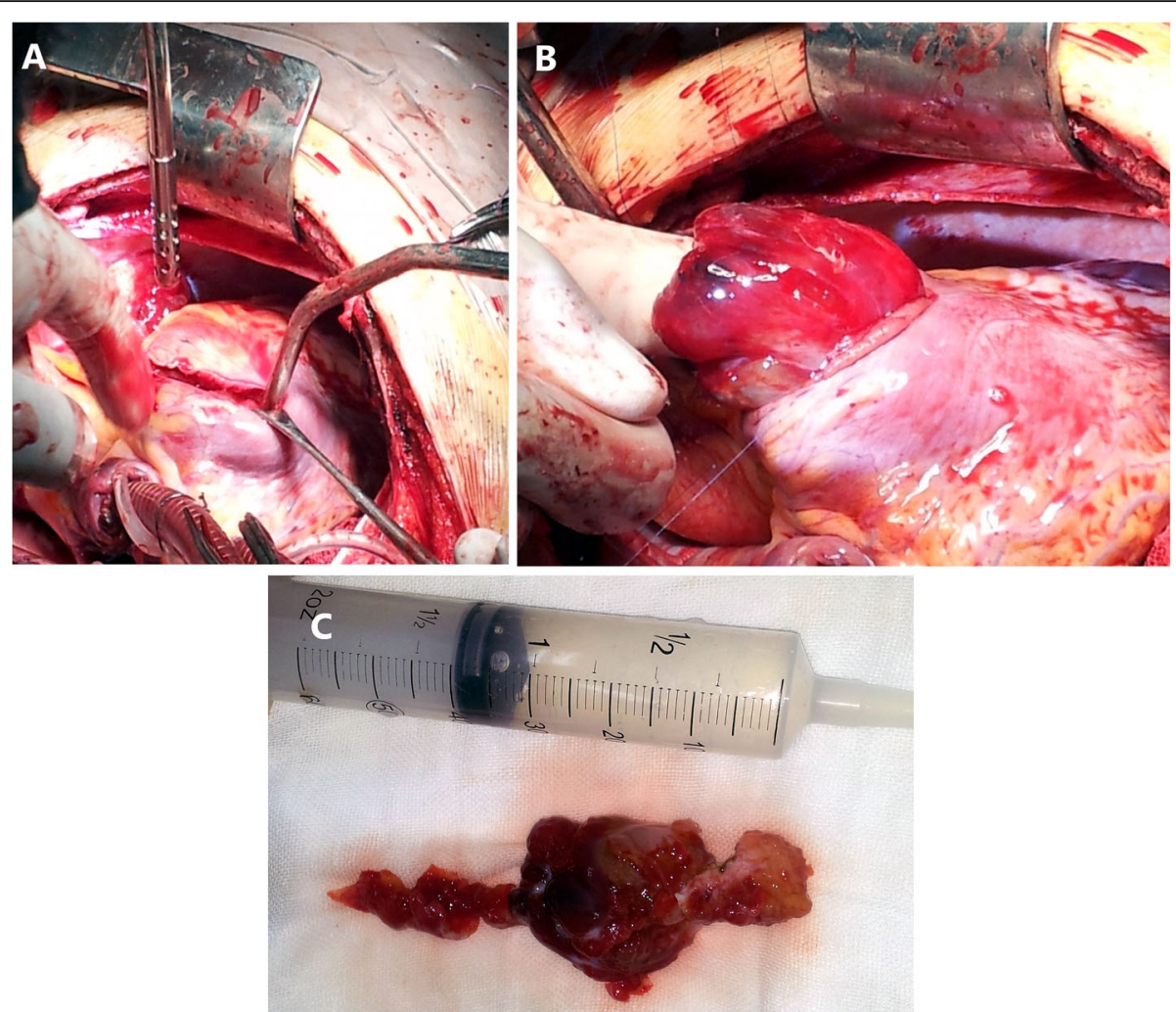

Fig. 5 Myxoma of the right ventricular outflow tract. a An 8-cm right ventriculotomy incision was at the infundibulum of the RVOT $2 \mathrm{~cm}$ lateral to the left anterior descending coronary artery. $\mathbf{b}$ The mass is shown out of the right ventriculotomy incision with its basal attachment to the IVS. c The specimen after excision from the IVS

Seventy-five percent of myxomas arise in the left atrium, $18 \%$ in the right atrium [2]. They rarely occur in the ventricles with a reported incidence of (3-4\%) [2].

Myxomas are pedicled in most cases and extend into the affected chamber. In life-threatening cases, the cavity can be filled by the tumor mass, and the tumor may prolapse to the ventricle during diastole, especially in latepresenting cases [2].

We presented a rare case that originated from the IVS that was obstructing the RVOT. Cardiac myxoma extending into the RVOT is rare, especially in early childhood. Symptoms and signs include peripheral edema, ascites, and shortness of breath as a result of RVOT obstruction $[3,7,9]$.

According to previous reports, LV myxomas usually arise from the septal endocardium [10], and there are a few case reports of growth from the LV free wall $[8,11]$. Cardiac myxomas related to the Carney complex (CNC) more frequently occur in the left ventricle, compared with sporadic myxomas [11].

Hemangioendothelioma is a rare vascular tumor that has unpredictable behavior $[12,13]$. To our best knowledge, the number of reported cases worldwide does not exceed 20 [12-15]. Mitral valve involvement in primary cardiac hemangioendothelioma is extremely rare, with three cases reported in the literature [12, 13].

Based on the WHO classification, hemangioendothelioma is classified into benign, intermediate (locally aggressive), or malignant tumors [16, 17].

Hemangioendothelioma occurs in nearly all age groups and shows no sex predilection [16]. However, tumors developing within the heart of infants are extremely rare. To date, there is one infant reported in the literature by Seo, and associates [16] for a half monozygotic twin [16] who presented with an upper respiratory tract infection.

The reported sites of hemangioendothelioma are the left atrium [12-15, 18, 19], mitral valve [12, 13], right atrium with or without extension to the superior venacava $[13,16,20,21]$. Both local invasion and distant metastasis were reported in some cases [21].

Treatment of this type of tumor includes wide surgical resection, radiotherapy, and chemotherapy, and the reported survival was up to 3 years [12, 13, 15]. Studies have reported difficulty in achieving complete resection of tumors with negative margins. In cases in which complete resection is possible, patients commonly experience local recurrence [12, 15, 19]. Our study presented a case of hemangioendothelioma who was 
managed with complete resection and had a recurrence after 1 year.

Timely detection and a radical surgical resection is, so far, the more acceptable management, given the limited experience for handling this type of tumor [12-19]. Our experience suggests an aggressive clinical approach and surgical removal within the first hours of the suspected diagnosis for the greatest chance of complete resection and reducing the risk of recurrence.

Primary cardiac lymphoma (PCL) is one of the rarest tumors of the heart. It belongs to extranodal nonHodgkin's lymphomas [22]. It composes $1.3 \%$ of all cardiac tumors and $0.5 \%$ of all extranodal lymphomas [22]. The most common type of tumor is diffuse large B cell lymphoma [23].

The right atrium [24] and ventricle are usually involved [25], and biatrial cases were reported [26]. Rare sporadic PCLs were reported in the left ventricle [27]. Treatment is a combination of surgery and chemotherapy [23]. We report a case of primary cardiac lymphoma of the left atrium.

Post-treatment surveillance for PCL should include FDGPET/CT scanning because relapses are common [22].

A review by Petrich and coworkers [28], including 197 cases of PCL published between 1947 and 2009, found that it is more common in the elderly age (median age 63 years). Ermann and coworkers [29] reported a series of 309 patients diagnosed with PCL between 2004 and 2015 , and the median survival was 49.5 months. The majority of PCL patients were male (54.7\%) and white (81.2\%), with a mean age of 68.3 years.

Chen and associates [23] reported 101 cases in a systematic review of literature from 2009 to 2019. They concluded that surgery alone did not improve patients' long-term prognosis or reduce the risk of death within 1 month [23].

Pediatric primary cardiac tumors in children are mostly benign, while approximately $10 \%$ are malignant [3]. Cardiac rhabdomyoma is the most common cardiac tumor during fetal life and childhood (40-60\%). Myxoma is exceedingly rare in fetuses and neonates [3]. We present three cases of pediatric cardiac tumors: an extremely rare case of infantile right atrial myxoma in a 2month-old infant and 2 cases of intracardiac rhabdomyoma aged 3 months and 1 year.

Surgical modalities vary according to the site and the size of the tumor. Cardiac tumors are challenging for cardiac surgeons because countless adaptability is vital. The heart team must be highly experienced in adult, pediatric, and rhythm surgery, as well as transplantation and artificial heart implantation [2].

Simple resection is the treatment of choice for benign tumors such as myxomas. Attention is crucial in cannulation and bypass to circumvent dislodging fragment.
We open both atria from the right superior pulmonary vein without injuring the tumor. The tumor and its root can then be removed from the septum en block. All the chambers of the heart should be inspected to exclude the presence of further tumors.

Complex tumor resection is conceivable, providing the mass is limited to the heart and, if the malignant tumor has not infiltrated the adjacent tissues.

In advanced tumors, including the right side of the heart, the whole right half can be resected, and pulmonary blood flow is secured utilizing Fontan circulation; however, right heart failure may result [18].

Our results designate that the results of surgical treatment of benign cardiac tumors are excellent in all age groups, with a meager rate of recurrence. The long-term outcomes are favorable. For malignant cardiac tumors, however, the literature reports a dismal prognosis [8]. Survival rates vary from 7 months to a maximum of 2 years from diagnosis $[6,8]$. On long-term follow-up, the majority of patients die of distant metastases. The median survival time of patients treated conventionally with chemotherapy and radiotherapy alone is less than a year $[8,15,30]$.

Simple, incomplete resection (debulking) outspreads survival by only a few months [5]. Radical resection combined with postoperative chemotherapy improves the long-term outcome [9]. Heart transplantation remains an option for extensive cardiac tumors with better survival rates [30].

\section{Study limitations}

Although the study is prospective multicenter research, myxomas were the main bulk of cases encountered. Mid-term and long-term survival are lacking. Left ventricular assist devices are not available at our centers nor heart transplantation. A larger sample is needed to include more pathologies, and mid-term follow-up is required to assess morbidity and mortality.

\section{Conclusions}

The diagnosis of cardiac tumors is challenging. The clinical scenario depends mainly on tumor location and size rather than the histopathology. Advanced imaging modalities are rarely required in primary cases, and echocardiography is the main diagnostic modality. Surgical resection has a good prognosis in benign tumors, while it is very challenging in malignant tumors, and multimodal therapy is required.

\section{Abbreviations}

MRI: Magnetic resonance imaging; CT: Computed tomography; IVC: Inferior vena cava; RA: Right atrium; NYHA: New York Heart Association Dyspnea Classification; TEE: Transesophageal echocardiography; TTE: Transthoracic echocardiography; IAS: Interatrial septum; AML: Anterior mitral leaflet; LCA: Left Circumflex Artery; MVR: Mitral valve replacement; ICU: Intensive care unit; CMRI: Cardiac magnetic resonance imaging; FDG-PET-CCT: 18F- 
fluorodeoxyglucose positron emission tomography (PET-CT); LVEF: Left ventricular function; SUVmax: Maximum standard uptake value; LVOT: Left ventricular outflow tract; LA: Left atrium; LV: Left ventricle; IV: Intravenous; RVOT: Right ventricular outflow tract; IVS: Interventricular septum; LAD: Left anterior descending coronary artery; MDT: Multidisciplinary team; CNC: Carney complex; PCL: Primary cardiac lymphoma; NHL-DLBCL: NonHodgkin's lymphoma, diffuse large beta-cell lymphoma

\section{Acknowledgements}

This trial would not be possible without the enthusiasm and commitment of clinicians, nurses, and our patients. The authors would like to thank Dr. Hatem Beshir https://orcid.org/0000-0003-1366-3949 from the department of cardiothoracic surgery at Mansoura University for his tremendous efforts in literature review, analyzing the data, writing, and revision of this manuscript.

\section{Previous presentations}

Early results were presented as an oral presentation during the proceedings of the 25th annual meeting of the Egyptian Society of Cardiothoracic Surgery, April 2018 in Cairo, Egypt.

Presented as a poster presentation \#470 during the proceedings of the 26th annual meeting of the Asian Society for Thoracic and Cardiovascular Surgery (ASCVTS 18), May 2018 in Moscow, Russia.

Results presented orally at the 15th annual congress of update in cardiology and cardiovascular surgery (UCCVS 2019), March 2019 in Antalya, Turkey. (conference abstract only was published in American Heart journal). One case scenario was presented as an independent manuscript. Arafa S, Sanad M. Primary cardiac hemangioendothelioma of the mitral valve. Egypt Heart J. 2018 Sep;70(3):229-232. doi: 10.1016/j.ehj.2018.06.002. Epub 2018 Jul 7. PMID: $30190651 ;$ PMCID: PMC6123280.

\section{Authors' contributions}

All authors contributed equally in conceptualization. MS, WA, and MAH operated the cases. MS, WA, and MAH performed the literature review. SA performed echocardiographic assessment for all cases and participated in writing of the manuscript with MS and WA. MS and WA analyzed the results. All the authors have read and approved the manuscript in its currently submitted form.

\section{Funding}

No funding was received, self-funded.

\section{Availability of data and materials}

The datasets used and/or analyzed during the current study are available from the corresponding author on reasonable request.

\section{Ethics approval and consent to participate}

We followed the declaration of Helsinki regarding studies on human subjects. Approvals of the institutional research boards of Mansoura University Faculty of Medicine (MUIRB-207-9-16) and Nasser Institute of Research and Treatment (NIRT-208-7-16) were obtained. Patients were informed and signed written consents for participation in the study, operation, and publishing of clinical data.

\section{Consent for publication}

Patients were informed and signed written consents for publishing of clinical data.

\section{Competing interests}

None to declare

\section{Author details}

'Department of Cardiothoracic Surgery, Faculty of Medicine, Mansoura University, Mansoura, Dakahlia, Egypt. ${ }^{2}$ Department of Cardiothoracic Surgery, Nasser Institute for Research and Treatment, Egypt Ministry of Health and Population, Cairo, Egypt. ${ }^{3}$ Department of Cardiothoracic Surgery, Mansoura International Hospital, Egypt Ministry of Health and Population, Mansoura, Dakahlia, Egypt. ${ }^{4}$ Department of Cardiology and Angiology, Faculty of Medicine, Mansoura University, Mansoura, Dakahlia, Egypt. ${ }^{5}$ Department of Anesthesia and Surgical intensive care, Faculty of Medicine, Mansoura University, Mansoura, Dakahlia, Egypt.
Received: 29 April 2020 Accepted: 17 May 2020

Published online: 16 June 2020

\section{References}

1. Ren DY, Fuller ND, Gilbert SAB, Zhang Y (2017) Cardiac tumors: clinical perspective and therapeutic considerations. Curr Drug Targets 18(15):18051809

2. He DK, Zhang YF, Liang Y, Ye SX, Wang C, Kang B, Wang ZN (2015) Risk factors for embolism in cardiac myxoma: a retrospective analysis. Med Sci Monit 21:1146

3. Uzun O, Wilson DG, Vujanic GM, Parsons JM, De Giovanni JV (2007) Cardiac tumours in children. Orphan J Rare Dis 2(1):11

4. Kumar N, Agarwal S, Ahuja A, Das P, Airon B, Ray R (2011) Spectrum of cardiac tumors excluding myxoma: experience of a tertiary center with review of the literature. Pathol Res Pract 207:769-774

5. Wang Z, Chen S, Zhu M, Zhang W, Zhang H, Li H, Yuan G, Zou C (2016) Risk prediction for emboli and recurrence of primary cardiac myxomas after resection. J Cardiothorac Surg 11(1):22

6. Li S, Gao C (2017) Surgical experience of primary cardiac tumor: singleinstitution 23-year report. Med Sci Monit 23:2111

7. Nomoto N, Tani T, Konda T, Kim K, Kitai T, Ota M, Kaji S, Imai Y, Okada Y, Furukawa Y (2017) Primary and metastatic cardiac tumors: echocardiographic diagnosis, treatment and prognosis in a 15-years single center study. J Cardiothorac Surg 12(1):103

8. Mahmoud HM, Moursi I (2014) A rare case of a big left ventricular myxoma presenting with a cerebrovascular stroke. Egypt Heart J 66(4):375-377

9. Hoffmeier A, Sindermann JR, Scheld HH, Martens S (2014) Cardiac tumors - diagnosis and surgical treatment. Dtsch Arztebl Int 111(12):205

10. Fakhari S, Bilehjani E (2016) A large left ventricle myxoma: presenting with epigastric pain and weight loss. Case Rep Cardiol 2016

11. Kuyama N, Hamatani $Y$, Fukushima S, Ikeda Y, Nakai E, Okada A, Takahama H, Amaki M, Hasegawa T, Sugano Y, Kanzaki H, Fujita T, Ishibashi-Ueda H, Yasuda S, Anzai T, Kobayashi J (2018) Left ventricular myxoma with carney complex. ESC Heart Fail 5(4):713-715

12. Arafa S, Sanad M (2018) Primary cardiac hemangioendothelioma of the mitral valve. Egypt Heart J 70(3):229-232

13. Ellouze M, Dami M, Beaulieu Y, Perrault LP, Romeo P (2015) Resection of a right atrial epithelioid hemangioendothelioma. Cardiovasc Pathol 24(6):401404

14. Cottini M, Lappa A, Donfrancesco S. Primary cardiac hemangioendothelioma in the adult and cardiac involvement in patient with non-cardiac hemangioendothelioma. Review of the Literature. Cancer Stud Ther J Volume. 2017;2(5):1-6.

15. Safirstein J, Aksenov S, Smith F (2007) Cardiac epithelioid hemangioendothelioma with 8-year follow-up. Cardiovasc Pathol 16(3):183186

16. Seo JW, Song MK, Park SH, Park HE, Park SA (2019) Primary cardiac hemangioendothelioma in an infant: a case report. Clin Pediatr Hematol Oncol 26:60-65

17. Bartsch $\mathrm{H}$, Haugen $\mathrm{A}$, Risch A. World Health Organization classification of tumours. Pathology and Genetics of Tumours of the Lung, Pleura, Thymus and Heart. Chapter 1, Tumours of the Lung. 2004:24Ǧ25. [Online]: available from Url: https://patologi.com/who\%20lunge.pdf

18. Lisy M, Beierlein W, Müller H, Bültmann B, Ziemer G (2007) Left atrial epithelioid hemangioendothelioma. J Thorac Cardiovasc Surg 133(3):803-804

19. Marchiano D, Fisher F, Hofstetter S (1993) Epithelioid hemangioendothelioma of the heart with distant metastases. A case report and literature review. J Cardiovasc Surg 34(6):529-533

20. Allain G, Hajj-Chahine J, Lacroix C, Tomasi J, Corbi P, Jayle C (2014 Nov) Surgical management of an epithelioid hemangioendothelioma of the superior vena cava protruding into the right atrium. J Card Surg 29(6):779781

21. Ishibashi H, Takasaki C, Akashi T, Okubo K. Successful excision of epithelioid hemangioendothelioma of the superior vena cava. The Annals of thoracic surgery. 2020 Apr(4):e271-e273. [svc\}

22. Jonavicius K, Salcius K, Meskauskas R, Valeviciene N, Tarutis V, Sirvydis V (2015) Primary cardiac lymphoma: two cases and a review of literature. J Cardiothorac Surg 10(1):138

23. Chen H, Qian S, Shi P, Liu L, Yang F (2020) A presentation, treatment, and survival analysis of primary cardiac lymphoma cases reported from 2009 to 2019. Int J Hematol 13:1-9 
24. Yousif P, Kotecha A, Thakur A, Ismail HM (2019) A rare case of diffuse large B cell lymphoma presenting as a cardiac mass. Am J Case Rep 20:182

25. Tabbah R, Nohra E, Rachoin R, Saroufim K, Harb B (2020) Lymphoma involving the heart: a case report. Front Cardiovasc Med 7:27

26. Li Y, Zhou Z, Xin F, Zhang C, Zhang R, Sun D, Liu W (2019) Primary cardiac lymphoma in both atria: a case report. J Clin Ultrasound 47(9):561-563

27. Matsunaga K, Kobayashi T, Takahashi M, Gohra H. A huge primary cardiac malignant lymphoma in the left ventricle. The Annals of Thoracic Surgery. 2020.

28. Petrich A, Cho SI, Billett H (2011) Primary cardiac lymphoma. Cancer. 117: 581-589

29. Ermann DA, Noble W, Parekh JD, Kapoor V, Latif A, Silberstein PT. Primary cardiac lymphoma: characteristics, treatment, and survival utilizing the National Cancer Database (NCDB). InB69. THORACIC ONCOLOGY: OPTIMIZING DIAGNOSIS AND MANAGEMENT 2019 May (pp. A3990-A3990). American Thoracic Society.

30. Hoffmeier A, Deiters S, Schmidt C, Tjan TD, Schmid C, Drees G, Fallenberg EM, Scheld HH (2004) Radical resection of cardiac sarcoma. Thorac Cardiovasc Surg 52(02):77-81

\section{Publisher's Note}

Springer Nature remains neutral with regard to jurisdictional claims in published maps and institutional affiliations.

\section{Submit your manuscript to a SpringerOpen ${ }^{\circ}$ journal and benefit from:}

- Convenient online submission

- Rigorous peer review

- Open access: articles freely available online

- High visibility within the field

- Retaining the copyright to your article

Submit your next manuscript at $\boldsymbol{\nabla}$ springeropen.com 